\title{
Note on Betti numbers of Riemannian manifolds III.
}

\author{
By Yasuro Tomonaga.
}

(Received July 27, 1953)

In continuation of our former papers, ${ }^{1)}$ we consider a Riemannian manifold which is compact orientable and whose fundamental differential form is positive definite. Consider an infinitesimal motion

$$
\bar{x}^{i}=x^{i}+\varepsilon X^{i},
$$

where $X^{i}$ satisfies Killing's equations

$$
X_{i ; j}+X_{j ; i}=0 \text {. }
$$

It follows from (2) that

$$
X_{i ; j ; k}+R_{i j k l} X^{l}=0 .
$$

If $X^{i}$ satisfies only (3), the transformation (1) defines an infinitesimal affine collineation.

From (3) we have

$$
\Delta X^{i}=-R^{i}{ }_{j} X^{j} .
$$

Consider a symmetric tensor $A_{i j}$ whose quadratic form

$$
A_{i j} f^{i} f^{i}
$$

is positive definite. Then we get by the same way as before

$$
-\int\left(\frac{1}{2} \Delta A_{i j}+A_{i k} R_{j}^{k}\right) X^{i} X^{j} d v+\int A_{i j} X^{i, k} X_{; k}^{j} d v=0 .
$$

Hence, if the equadratic form

$$
Q=\left(\frac{1}{2} \Delta A_{i j}+A_{i k} R_{j}^{k}\right) f^{i} f^{j}
$$

is everywhere negative definite, it follows from (5) that

$$
X^{i}=0,
$$

1) Note on Betti numbers of Riemannian manifolds I, II, Jour. Math. Soc. Japan, 5 (1953), 59-69. 
i. e. our manifold admits no infinitesimal motion (affine collineation). If $Q$ is everywhere negative semi-definite, we have

$$
X_{i ; j}=0 \text {, }
$$

hence the vector $X^{i}$ contains at most $n$ arbitrary constants.

Especially in the case in which

$$
A_{i j}=\rho^{2} g_{i j} \quad(\rho \neq 0)
$$

we have

$$
\begin{aligned}
Q & =\frac{1}{2}\left(\Delta \rho^{2}\right) f_{i} f^{i}+\rho^{2} R_{i j} f^{i} f^{j} \\
& \leqq\left\{\frac{1}{2}\left(\Delta \rho^{2}\right)+\frac{R}{n} \rho^{2}+\rho^{2} \sqrt{R_{a b} R^{a b}-\frac{R^{2}}{n}}\right\} f_{i} f^{i}
\end{aligned}
$$

Hence, if there exists a scalar such that the inequality

$$
\frac{1}{2}\left(\frac{\Delta \rho^{2}}{\rho^{2}}\right)+\frac{R}{n}+\sqrt{R_{a b} R^{a b}-\frac{R^{2}}{n}}<0
$$

holds everywhere, then our manifold admits no infinitesimal motion (affine collineation). If it holds that

$$
\frac{1}{2}\left(\frac{\Delta \rho^{2}}{\rho^{2}}\right)+\frac{R}{n}+\sqrt{R_{a b} R^{a b}-\frac{R^{2}}{n}} \leqq 0,
$$

then $X^{i}$ satisfies

Especially in the case

$$
X_{i: j}=0 \text {. }
$$

$$
\rho^{2}=1+c^{2} R^{2}
$$

we have the

THEOREM 1. If the inequality

$$
\frac{1}{2}\left(\frac{c^{2} \Delta R^{2}}{1+c^{2} R^{2}}\right)+\frac{R}{n}+\sqrt{R_{a b} R^{a b}-\frac{R^{2}}{n}}<0
$$

holds everywhere for a certain constant $c$, our manifold admits no infinitesimal motion (affine collineation). If

$$
\frac{1}{2}\left(\frac{c^{2} \Delta R^{2}}{1+c^{2} R^{2}}\right)+\frac{R}{n}+\sqrt{R_{a b} R^{a b}-\frac{R^{2}}{n}} \leqq 0
$$


holds, then $X^{i}$ satisfies

$$
X_{i ; j}=0 \text {. }
$$

By the same way, we have, for Betti numbers, the THEOREM 2. If the inequality

$$
\frac{1}{2}\left(\frac{c^{2} \Delta R^{2}}{1+c^{2} R^{2}}\right) \leqq \frac{R}{n}-\sqrt{R_{a b} R^{a b}-\frac{R^{2}}{n}}
$$

holds everywhere for a certain constant $c$, then we have

$$
B_{1} \leqq n \text {. }
$$

If, in (13), the equality sign can be omitted, then we have

$$
B_{1}=0 \text {. }
$$

THEOREM 3. If the inequality

$$
\begin{aligned}
& \frac{1}{2}\left(\frac{c^{2} \Delta R^{2}}{1+c^{2} R^{2}}\right) \leqq p\left\{\frac{n-p}{n(n-1)} R\right. \\
& -\sqrt{\left.\left(\frac{p-1}{2}\right)^{2} R_{i j k l} R^{i j k l}+\frac{n-4 p+2}{4} R_{i j} R^{i j}+\left\{\frac{1}{4}-\frac{(n-p)^{2}}{2 n(n-1)}\right\} R^{2}\right\}}
\end{aligned}
$$

holds everywhere for a certain constant $c$, it holds that

$$
B_{p} \leqq\left(\begin{array}{c}
n \\
p
\end{array}\right)
$$

and the covariant derivative of any harmonic tensor of degree $p$ vanishes. If, in (14), the equality sign can be omitted, then we have

$$
B_{p}=0 \text {. }
$$

Utunomiya University. 